\title{
ESTIMANDO A FRAÇÃO DE FASES EM UM AÇO MULTIFÁSICO ATRAVÉS DE MEDIDAS DE SATURAÇÃO MAGNÉTICA
}

\author{
Daniel Fevereiro Valdebenito ' \\ Arthur Seiji Nishikawa' \\ Marcelo Shiroma Lancarotte ${ }^{2}$ \\ Hélio Goldenstein ${ }^{3}$
}

\section{Resumo}

O uso crescente de aços multifásicos com o objetivo de obter simultaneamente melhor resistência e melhor tenacidade tem aumentado a demanda por técnicas de caracterização ao mesmo tempo rápidas e baratas para identificar e quantificar as fases presentes na microestrutura. Um método muito comum e preciso para esta tarefa é a análise por Difração de Raios-X (DRX), que necessita equipamento sofisticado e pessoal especializado, nem sempre disponível fora do ambiente acadêmico. O objetivo do presente trabalho é comparar a técnica convencional por DRX com resultados obtidos por medidas de saturação magnética, na identificação e quantificação das frações das fases presentes em amostras de um aço experimental baseado no aço para rolamentos AISI 52100. As amostras foram austenitizadas no campo intercrítico e tratadas isotermicamente usando um dilatômetro de têmpera. As medidas de saturação magnética utilizaram um equipamento especialmente projetado, o MagSat, construído pela Globalmag Transdutores Magnéticos Ltda. A saturação magnética encontrada foi comparada ao valor da saturação magnética do ferro puro, sendo a diferença proporcional à fração de fases paramagnéticas.

Palavras-chave: Aços multifásicos; Saturação magnética; Fração de fases.

\section{ESTIMATING PHASE FRACTION IN A MULTIPHASE STEEL THROUGH MEASURES OF SATURATION MAGNETIZATION}

\begin{abstract}
The increasing use of multiphase steels so as to simultaneously optimize strength and toughness, multiplied the demand for quick and cheap characterization techniques able to identify and quantify the phases in the microstructure. A common and precise method is X-Ray Diffraction (XRD), which requires sophisticated equipment and trained personnel though, not always available outside the academic environment. The objective of this study is to compare conventional technique by XRD with results obtained by magnetic saturation measurements, applied to the identification and quantification of the phases fractions in samples from an experimental bearing steel, similar to AISI 52100. Samples were austenitized in the intercritical field and isothermally treated using a quenching dilatometer. The magnetic saturation experiments were made using specially designed equipment, the MagSat, custom built by Globalmag Transdutores Magnéticos Ltda. The measured magnetic saturation was compared to the value of the magnetic saturation of pure iron, the difference being proportional to the fraction of paramagnetic phases.
\end{abstract}

Keywords: Multiphase steels; Saturation magnetization; Phases fraction.

\section{INTRODUÇÃO}

Tradicionalmente, os aços estruturais tinham estruturas de ferrita e perlita, enquanto os aços de construção, temperados e revenidos, tinham uma estrutura predominante de martensita e carbonetos. Essas últimas estruturas conferem ao aço dureza e boa resistência mecânica, porém, pouca resistência ao impacto e ductilidade. Neste contexto, há

'Escola Politécnica, Universidade de São Paulo - USP, São Paulo, SP, Brasil. E-mail: danferval@gmail.com

${ }^{2}$ GlobalMag Transdutores Magnéticos Ltda., Cotia, SP, Brasil.

${ }^{3}$ Departamento de Engenharia Metalúrgica e de Materiais, Escola Politécnica, Universidade de São Paulo - USP, São Paulo, SP, Brasil. 
alguns anos, novas gerações de aços foram desenvolvidas para aplicações estruturais, como os aços com efeito TRIP, aços Dual-Phase, aços Maraging, aços de alta e ultra alta resistência (AHSS e UHSS). Novos tratamentos térmicos também foram sendo desenvolvidos, como a têmpera e lamelarização, austêmpera e a têmpera e partição (Q\&P) [I,2]. Nestes tratamentos, a bainita é geralmente o constituinte mais importante, em geral associado a pequenas quantidades de austenita retida, isolada ou na forma de agregados de austenita e martensita (microconstituinte MA). Na obtenção destes aços multifásicos é bastante conhecido o efeito dos elementos de liga Si e Al, que inibem a precipitação de cementita no resfriamento pós austenitização $[1,3,4]$. Um dos produtos possíveis é a bainita isenta de carbonetos, "carbide-free bainite". Além deste, Caballero e Bhadeshia [5] descreveram ligas e tratamentos onde foi obtido um constituinte bainítico super refinado, chamado de nanobainita. Na grande maioria destes estudos, os aços estudados são submetidos inicialmente a uma austenitização plena [6].

Em trabalhos anteriores, os presentes autores empregaram um aço baseado no aço AISI 52100 [7], com $\mathrm{Mn}$ e Si incrementados, submetendo-o a tratamentos de austêmpera com austenitização no campo intercrítico [8]. O resultado foi um material multicomponente, bainítico contendo também carbonetos primários. Devido ao alto teor de $\mathrm{Si}$, a formação da cementita foi inibida, sobrando maior quantidade de $\mathrm{C}$ para ser particionado para a austenita durante o resfriamento, ficando assim a estrutura final com cementita e bainita, e teores significativos de austenita retida enriquecida em $\mathrm{C}$, estável à temperatura ambiente.

O método mais comum para identificação das fases e suas frações é a Difração de Raios-X (DRX). Porém, o método apresenta alguns inconvenientes, como a necessidade de profissionais especializados, resultado de difícil interpretação, eventual sobreposição de picos, ruído de fundo, difícil preparação da amostra, possível contaminação da amostra ou do equipamento, custo elevado, etc. A identificação da austenita retida também pode ser prejudicada por efeitos da textura (orientação). Desta forma, o desenvolvimento de outras técnicas de identificação e quantificação de fases em aços é útil e desejável. A literatura menciona algumas técnicas de medição magnética das fases $[9,10]$.

O Magnetômetro de Amostra Vibrante (VSM) é o magnetômetro mais utilizado em laboratórios de pesquisa, seja por seu baixo custo ou por sua simplicidade de funcionamento.
Consiste em um elemento vibrante tipo alto-falante que faz a amostra vibrar a uma determinada frequência dentro de um campo magnético. Bobinas de detecção são induzidas por uma tensão alternada de mesma frequência. $O$ sinal é tratado e interpretado.

Ferritoscópio: É um equipamento que mede o teor de ferrita em uma amostra, com base no teor de fase ferromagnética. Ele se baseia nos princípios de indução e susceptibilidade magnética. Uma bobina gera um campo magnético conhecido, que interage com as fases da amostra. Uma segunda bobina detecta as mudanças no campo após esta interação. A queda no campo magnético é proporcional à interação com fases paramagnéticas, podendo assim, medir-se suas frações.

Saturação Magnética: é o estado alcançado quando a magnetização de um material não pode mais ser aumentada por adição de um campo magnético externo. Neste momento, todos os domínios magnéticos estão alinhados. Sabe-se por medições, que a ferrita atinge, quando saturada magneticamente por um campo magnético forte, magnetização de 2, I5T (Tesla) [10]. Assim, usando o mesmo princípio do ferritoscópio, é possível mensurar frações de fases de um material. Para aços multifásicos, no entanto, técnicas auxiliares podem ser necessárias.

No presente trabalho, será empregado o efeito da saturação magnética para identificar as fases e quantificar suas frações presentes em amostras de um aço baseado no AISI 52100 , em um equipamento desenvolvido especialmente para ensaios em amostras provenientes de tratamentos em dilatômetro. Os resultados serão comparados aos resultados obtidos por Difração de Raios-X.

\section{METODOLOGIA}

\section{I Materiais}

Uma das ligas foi desenvolvida com base no aço para rolamentos AISI 52100, tendo os teores de $\mathrm{Si}$ e Mn incrementados, conforme Tabela I. A barra foi produzida em forno de fusão por indução a vácuo, no centro de pesquisa da Villares Metals S.A. em Sumaré, SP. Para os corpos de prova padrão para aferição do equipamento de saturação magnética, foi usado um aço para tubo API X65 comercial, descrito na Tabela 2.

Tabela I. Composição química (\%) do material estudado e do material comercializado

\begin{tabular}{ccccccccccccc}
\hline Aço & $\mathbf{C}$ & $\mathbf{S i}$ & $\mathbf{M n}$ & $\mathbf{P}$ & $\mathbf{S}$ & $\mathbf{C r}$ & $\mathbf{N i}$ & $\mathbf{M o}$ & $\mathbf{C u}$ & $\mathbf{A l}$ & $\mathbf{S n}$ & $\mathbf{O}_{2}$ \\
\hline $52100 \mathrm{com}$ & $\mathrm{I}, 00$ & 0,25 & 0,32 & 0,006 & 0,0056 & 1,35 & 0,04 & 0,01 & 0,01 & 0,019 & - & - \\
$52100 \mathrm{mod}$ & $\mathrm{I}, 06$ & $\mathrm{I}, 74$ & 0,96 & 0,002 & 0,001 & 1,49 & 0,13 & 0,05 & 0,06 & 0,05 & 0,006 & 0,0006 \\
\hline
\end{tabular}

Fonte: Adaptado de Marcomini e Goldenstein [7].

Tabela 2. Composição química para aço para tubo API X65 utilizado no MagSat

\begin{tabular}{ccccccccccccc}
\hline & $\mathbf{C}$ & $\mathbf{S i}$ & $\mathbf{M n}$ & $\mathbf{P}$ & $\mathbf{S}$ & $\mathbf{C r}$ & $\mathbf{N i}$ & $\mathbf{C u}$ & $\mathbf{T i}$ & $\mathbf{N b}$ & $\mathbf{A l}$ & $\mathbf{C a}$ \\
\cline { 2 - 12 } & $\%$ & $\%$ & $\%$ & $\mathbf{P p m}$ & $\mathbf{P p m}$ & $\%$ & $\%$ & $\%$ & $\%$ & $\%$ & $\%$ & $\mathbf{P p m}$ \\
\hline API X65 & 0,04 & 0,24 & 0,29 & 90 & 10 & 0,51 & 0,17 & 0,29 & 0,02 & 0,09 & 0,03 & 17 \\
\hline
\end{tabular}




\subsection{Métodos}

\subsection{Ensaios de dilatometria}

Foram preparadas as amostras da Tabela 3, com dimensão final de $\varnothing 4 \times 10 \mathrm{~mm}$. Estas amostras foram inicialmente retiradas de uma barra redonda de $\varnothing 50 \mathrm{~mm}$. A série $B$ foi retirada por seccionamento e torneamento. Posteriormente, as amostras da série $\mathrm{C}$ foram retiradas por eletroerosão a fio. Os cilindros foram retirados em ambos os casos axialmente, distante à superfície.

Estas amostras foram aquecidas em um dilatômetro de Têmpera BÄHR DIL805A até $865^{\circ} \mathrm{C}$ (campo $\Theta+\gamma$ ), permanecendo nesta temperatura por 30 minutos. Foram então resfriadas até diferentes temperaturas conforme a segunda coluna da Tabela 3 , acima e abaixo da temperatura Ms estimada em $208{ }^{\circ} \mathrm{C}$ pela equação de Andrews [II] (e confirmada pelos gráficos de dilatometria). As amostras permaneceram nestas temperaturas por 6 horas, conforme rotas de tratamento ilustradas na Figura I. As amostras da série $B$ foram submetidas a ensaios de DRX, e as amostras da série $C$ foram submetidas a ensaios de saturação magnética. As amostras TI e T2 foram austenitizadas da mesma forma que as demais, porém sofreram têmpera simples à temperatura ambiente e à $-196^{\circ} \mathrm{C}$ (no nitrogênio líquido), respectivamente. Elas também foram submetidas aos ensaios de DRX e de saturação magnética, como referência. A rota de tratamento foi estabelecida de modo obter-se uma estrutura de carbonetos primários e decomposição

Tabela 3. Amostras preparadas para tratamentos isotérmicos

\begin{tabular}{ccc}
\hline Amostra & Temperatura & Tempo \\
\hline $\mathrm{BI} / \mathrm{Cl}$ & $250^{\circ} \mathrm{C}$ & $6 \mathrm{~h}$ \\
$\mathrm{~B} 2 / \mathrm{C} 2$ & $230^{\circ} \mathrm{C}$ & $6 \mathrm{~h}$ \\
$\mathrm{~B} 3 / \mathrm{C} 3$ & $210^{\circ} \mathrm{C}$ & $6 \mathrm{~h}$ \\
$\mathrm{~B} 4 / \mathrm{C} 4$ & $200^{\circ} \mathrm{C}$ & $6 \mathrm{~h}$ \\
$\mathrm{~B} 5 / \mathrm{C} 5$ & $190^{\circ} \mathrm{C}$ & $6 \mathrm{~h}$ \\
$\mathrm{~B} 6 / \mathrm{C} 6$ & $180^{\circ} \mathrm{C}$ & $6 \mathrm{~h}$ \\
$\mathrm{TI}$ & $20^{\circ} \mathrm{C}$ & -- \\
$\mathrm{T} 2$ & $-196^{\circ} \mathrm{C}$ & - \\
\hline
\end{tabular}

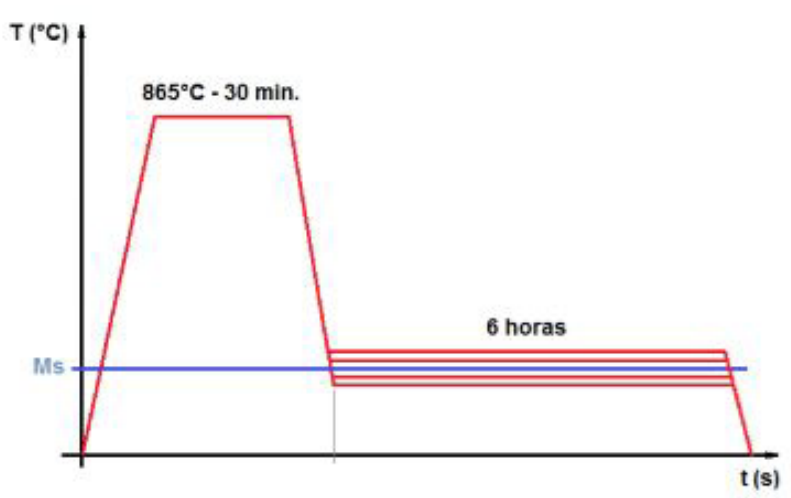

Figura I. Rotas de tratamento para as amostras $\mathrm{BI}$ a B6 e Cl a C6. isotérmica da austenita em uma estrutura multicomponente com diferentes frações de bainita livre de carbonetos, martensita e austenita retida enriquecida em C.

\subsubsection{Microscopia eletrônica de varredura (MEV-FEG)}

Ensaios executados em um MEV-FEG (Field Emission Electron Gun), marca FEI, modelo Inspect F50, feixe de $20 \mathrm{kV}$, detecção por elétrons secundários, aumentos de 5.000 a 100.000 vezes.

\subsubsection{Ensaios de difração de raios-X (DRX)}

Ensaios conduzidos nas amostras da Tabela 3 (série B) em um difratômetro PANalytical, modelo X'Pert PRO com detector X'Celerator. Foram utilizados: Tubo de $\mathrm{Cu}$, feixe de $40 \mathrm{kV}$, faixa angular $35-105^{\circ}(2 \Theta)$, passo angular $0,017^{\circ}$, com tempo/passo de 300s. O Refino dos picos foi feito pelo método de Rietveld utilizando-se o software GSAS. Apenas os picos da ferrita e da austenita foram refinados. Os picos da cementita foram desconsiderados devido à baixa intensidade e sobreposição com outros picos, o que gerava muita variação nos resultados.

\subsubsection{Ensaios de saturação magnética:}

Os ensaios de saturação magnética foram executados em um equipamento especialmente desenvolvido pela Globalmag Transdutores Magnéticos Ltda ilustrado na figura 2. Os procedimentos de condução das análises e ensaios foram especialmente elaborados e serão aqui descritos. Os ensaios foram conduzidos nas amostras da Tabela 3 (série C). $\mathrm{O}$ equipamento foi especialmente desenvolvido para amostras com dimensões de $\varnothing 4 \times 10 \mathrm{~mm}$, já que esta é a dimensão ideal para ensaios de dilatometria no dilatômetro de têmpera BÄHR DIL805A. As amostras são inseridas no equipamento e submetidas a um campo magnético $(H)$ fixo obtido com imãs de Fe-Nd-B com até $200 \mathrm{kA} / \mathrm{m}$, em contato direto com a amostra através de um sistema de "pole-piece" ajustável, ficando magneticamente saturadas. Uma bobina sonda envolvendo a amostra é ligada a um medidor de fluxo para monitorar a indução magnética (B).

Um computador faz a leitura do valor de saturação ( $\mathbf{s}$ ) em Tesla. Este valor é comparado com o valor de saturação de uma amostra de ferro puro $(2,15 T)$. A diferença entre os valores é proporcional à fração não magnética da amostra.
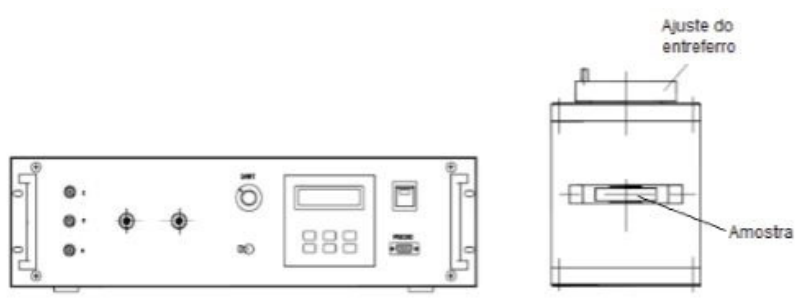

Figura 2. Representação esquemática do equipamento de Saturação Magnética. 
A austenita retida à temperatura ambiente é paramagnética. Já a cementita e a ferrita são ferromagnéticas. Porém, conforme descrito por Kagawa e Okamoto [12], o Cr diminui a temperatura de Curie da cementita, deixando-a paramagnética à temperatura ambiente para teor de $\mathrm{Cr}$ acima de 7,5\% (aproximadamente), conforme Figura 3. Para o material estudado, nas condições de equilíbrio a $865^{\circ} \mathrm{C}$, o teor de $\mathrm{Cr}$ na cementita, segundo simulações no ThermoCalc, é de 8,73\%. Ensaios de EDS foram conduzidos no MEV-FEG, para confirmar esta condição paramagnética da cementita. $\mathrm{O} M \mathrm{n}$ tem efeito similar. Uma vez obtido o valor de Js da amostra no equipamento, a Equação I pode ser utilizada para quantificação das fases. Nesta equação, têm-se " $\alpha$ " para microconstituintes ferríticos, " $\checkmark$ " para austenita e " $\Theta$ " para cementita.

$$
\mathbf{J s}=\mathbf{f}_{\alpha} \times \mathbf{J} \mathbf{s}_{\alpha}+\mathbf{f}_{x} \times J \mathbf{s}_{x}+\mathbf{f}_{\Theta} \times \mathbf{J} \mathbf{s}_{\Theta}
$$

$\mathrm{s}=$ polarização de saturação (valor medido pelo equipamento). $f_{0}=$ fração volumétrica da fase $\alpha, x$ e $\Theta$.

$\mathrm{Js}_{0}=$ polarização de saturação da fase $\alpha, x$ e $\Theta$.

A fração de $f_{\Theta}$ utilizada foi a fração volumétrica encontrada no método de quantificação de fase metalográfica $(6,875 \%)$.

A fração de $\mathrm{f}_{\alpha}$ é dada isolando-se o termo na Fórmula $\mathrm{I}$. A fração de $\mathrm{f}_{x}$ é dada pela Fórmula 2:

$$
\mathbf{f}_{\alpha}+\mathbf{f}_{x}+\mathbf{f}_{\Theta}=\mathbf{I} \text {. }
$$

O valor de $\mathrm{Js}_{X}$ e $\mathrm{Js}_{\Theta}$ é zero, pois estes constituintes são paramagnéticos.

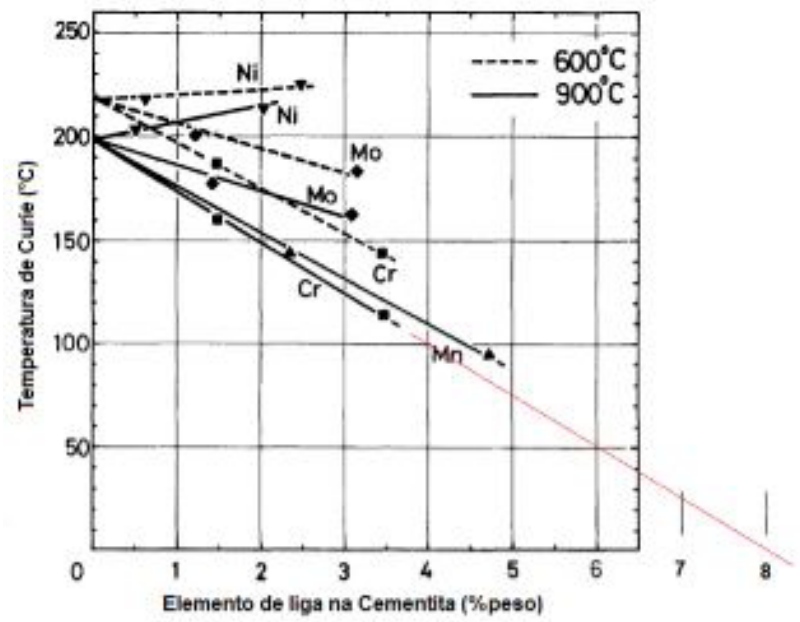

Figura 3. Variação na temperatura de Curie da cementita para ligas recozidas a 600 e $900{ }^{\circ} \mathrm{C}$ pelo teor dos elementos presentes na cementita. Fonte: Adaptado de Kagawa e Okamoto [12].
Já o valor de $\mathrm{Js}=1,924712 \mathrm{~T}$ foi calculado da seguinte forma:

A medida de 2,15T para saturação magnética da ferrita é teórica, e este valor necessita ser verificado experimentalmente no equipamento. Para isso, duas amostras (denominadas AI e A2) de Aço API X65, que, conforme Tabela 2, contém baixo carbono, foram retiradas de um tubo com costura, longe da região da solda, confeccionadas com as mesmas dimensões de $\varnothing 4 \times 10 \mathrm{~mm}$ e então ensaiadas. Após 10 medições (5 para cada amostra), o valor médio encontrado foi de 2,019T.

Foram então descontadas, conforme Equação 3, as porcentagens dos elementos de $\mathrm{Si}, \mathrm{Mn}, \mathrm{Cr}, \mathrm{Ni}$ e $\mathrm{Cu}$ das amostras $\mathrm{Al}$ e A2, pois estes elementos são paramagnéticos, chegando-se ao valor de $\mathrm{Js}_{\alpha}=2,049746 \mathrm{~T}$.

$\mathrm{JS}_{\mathrm{a}}=\frac{2,019}{(100-\% \mathrm{Si}-\% \mathrm{Mn}-\% \mathrm{Cr}-\% \mathrm{Ni}-\% \mathrm{Cu})} \times 100$

Este valor foi então multiplicado pela porcentagem atômica de ferro presente na austenita das amostras de AISI 52100 modificado. Foram, para tanto, estimados por ThermoCalc os teores de cada elemento do material AISI 52100 modificado para temperatura de $865^{\circ} \mathrm{C}$, conforme a Tabela 4. Subtraindo-se os valores de cada elemento na austenita de $100 \%$, encontramos o teor de ferro na austenita, o qual corrigindo para porcentagem atômica, obteve-se 93,89\%at. O valor final de Js $s_{\alpha}$ usado nos cálculos foi, portanto, Js $\mathrm{s}_{\alpha}=1,9247 \mathrm{I} 2 \mathrm{~T}$.

\subsubsection{Espectroscopia por Dispersão de Energia (EDS)}

Foi escolhida aleatoriamente a amostra B5 $\left(190^{\circ} \mathrm{C}-6 \mathrm{~h}\right)$ para verificação da quantidade de $\mathrm{Cr}$ na fase cementita através de Espectroscopia por Dispersão de Energia (EDS). Os parâmetros de ensaio estão na Tabela 5.

\subsubsection{Análise metalográfica quantitativa}

A fração de cementita das amostras foi estimada através de análise metalográfica quantitativa conforme norma ASTM E562 [13]. Foram utilizadas 20 imagens aleatórias das amostras obtidas por MEV-FEG, com aumentos de $5.000 \mathrm{e}$ 10.000 vezes, malha de $10 \times 10$ ( 100 pontos).

\section{RESULTADOS}

\section{I Microscopia Eletrônica por Varredura (MEV-FEG):}

Nas micrografias geradas por MEV-FEG encontrou-se a presença de carbonetos primários para todas as amostras. Grande quantidade de agulhas de bainita entremeadas por

Tabela 4. Porcentagem de cada elemento na cementita e na austenita, a $865^{\circ} \mathrm{C}$, estimado por ThermoCalc

\begin{tabular}{ccccccccc}
\hline \%peso & $\mathbf{C}$ & $\mathbf{S i}$ & $\mathbf{C r}$ & $\mathbf{M n}$ & $\mathbf{N i}$ & $\mathbf{C u}$ & $\mathbf{M o}$ & $\mathbf{A l}$ \\
\hline Cementita & 6,73 & 0,00 & 8,73 & $\mathrm{I}, 49$ & 0,02 & 0,00 & 0,22 & 0,00 \\
Austenita & 0,65 & 1,80 & 1,22 & 0,94 & 0,13 & 0,06 & 0,04 & 0,05 \\
\hline
\end{tabular}



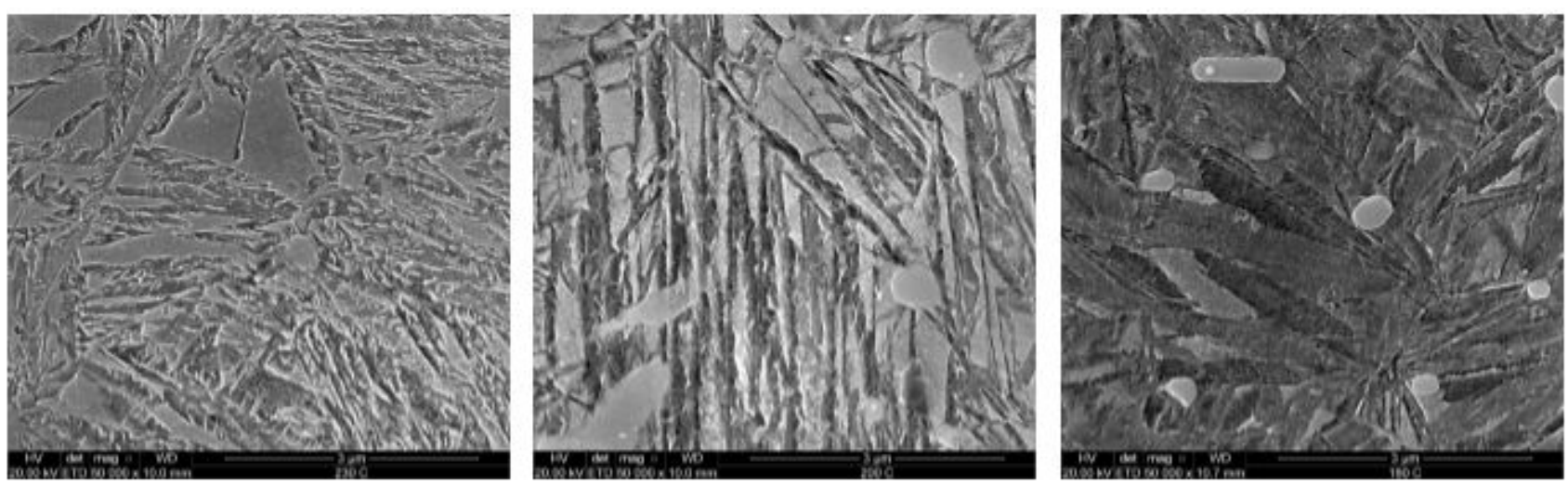

Figura 4. Micrografias MEV-FEG, da esquerda para direita: amostra B2 $\left(230^{\circ} \mathrm{C}-6 \mathrm{~h}\right)$, B4 $\left(200^{\circ} \mathrm{C}-6 \mathrm{~h}\right)$ e $\mathrm{B} 6\left(\mathrm{I} 80^{\circ} \mathrm{C}-6 \mathrm{~h}\right)$. Detecção por elétrons secundários.

Tabela 5. Parâmetros do ensaio de EDS

\begin{tabular}{ccc}
\hline Amostra & Feixe (kV) & Aumento \\
\hline B5 & 15 & $36.246 \times$ \\
\hline
\end{tabular}

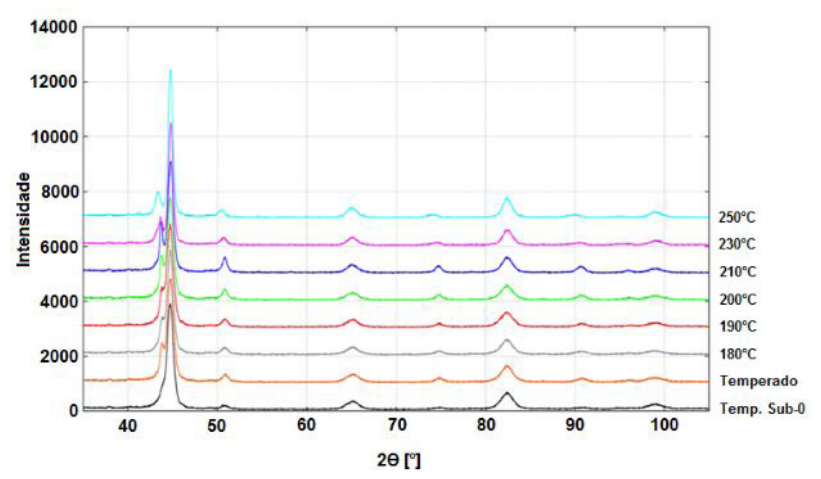

Figura 5. Difratogramas para as amostras BI a B6, TI e T2.

Tabela 6. Fração volumétrica para as amostras $\mathrm{BI}$ a B6, TI e T2, obtidas pelo GSAS por refino de Rietveld

\begin{tabular}{cccc}
\hline & $\begin{array}{c}\text { Fração vol. } \\
\text { Cementita }\end{array}$ & $\begin{array}{c}\text { Fração vol. } \\
\text { Ferrita }\end{array}$ & $\begin{array}{c}\text { Fração vol. } \\
\text { Austenita }\end{array}$ \\
\hline B6 $\left(180^{\circ} \mathrm{C}\right)$ & $6,875 \%$ & $75,76 \%$ & $17,36 \%$ \\
B5 $\left(190^{\circ} \mathrm{C}\right)$ & $6,875 \%$ & $76,83 \%$ & $16,29 \%$ \\
B4 $\left(200^{\circ} \mathrm{C}\right)$ & $6,875 \%$ & $68,41 \%$ & $24,71 \%$ \\
B3 $\left(210^{\circ} \mathrm{C}\right)$ & $6,875 \%$ & $63,58 \%$ & $29,54 \%$ \\
B2 $\left(230^{\circ} \mathrm{C}\right)$ & $6,875 \%$ & $75,85 \%$ & $17,28 \%$ \\
B1 $\left(250^{\circ} \mathrm{C}\right)$ & $6,875 \%$ & $76,71 \%$ & $16,42 \%$ \\
T1 $($ Têmpera $)$ & $6,875 \%$ & $74,24 \%$ & $18,89 \%$ \\
T2 (Têmpera Sub.) & $6,875 \%$ & $81,75 \%$ & $11,38 \%$ \\
\hline
\end{tabular}

austenita retida foi identificada para amostras $\mathrm{BI}$ e B2. Para as amostras B3 e B4, houve um aumento na quantidade de austenita retida, esta se apresentando em ilhas ou blocos poligonais, especialmente para amostra B3. Para as amostras B5 e B6, percebe-se a presença de martensita lenticular no lugar da bainita. Em todos os casos, no entanto, vemos as fases $\alpha$ (ferrita bainítica ou martensítica), $\chi$ (austenita retida) e $\Theta$ (cementita). A Figura 4 exemplifica a microestrutura encontrada em algumas amostras.

\subsection{Ensaios de Difração de Raios-X (DRX)}

A Figura 5 ilustra os difratogramas obtidos para as amostras BI a B6, TI e T2.

Com os dados fornecidos pelo software GSAS, pode-se calcular as frações volumétricas de cada constituinte, conforme Tabela 6 [8].

\subsection{Ensaios de Saturação Magnética}

Os ensaios de saturação magnética geraram os resultados apresentados na Tabela 7, aplicando-se as Fórmulas I e 2.

\subsection{Espectroscopia por Dispersão de Energia (EDS)}

A análise por EDS nos carbonetos primários da amostra $\mathrm{B} 5$ resultou em um valor de teor de $\mathrm{Cr}$ próximo ao teórico obtido no ThermoCalc de $8,73 \%$ peso, conforme apresentado na Tabela 8.

O valor encontrado, ligeiramente mais baixo que $o$ obtido por simulação, pode ser resultante de interação do feixe com a matriz ferrítica, uma vez que os carbonetos na amostra são muito pequenos. Para se obter resultados mais confíaveis, o feixe teve sua potência reduzida para $15 \mathrm{kV}$, causando assim menor interação com a matriz. É importante lembrar ainda que análises por EDS são semiquantitativas, e se considerarmos ainda o teor de $1,49 \%$ de $\mathrm{Mn}$ na cementita, a temperatura de Curie tende a ser ainda mais baixa. Assim, o valor foi considerado condizente com o valor teórico fornecido por simulação em software, e importante para confirmar o fenômeno da diminuição da temperatura de Curie nas análises de Saturação Magnética.

\subsection{Análise Metalográfica Quantitativa}

Os resultados do ensaio, conforme ASTM E562, estão na Tabela 9. 
Tabela 7. Determinação das frações de fases pelo método de Saturação Magnética para as amostras Cl a C6, TI e T2

\begin{tabular}{|c|c|c|c|c|c|c|c|c|c|c|c|c|c|}
\hline Amostra & Js & $=$ & $\mathbf{f}_{\alpha}$ & $x$ & Js ${ }_{\alpha}$ & + & $\mathbf{f}_{x}$ & $x$ & $\mathbf{J} \mathbf{s}_{x}$ & + & $\mathbf{f}_{\Theta}$ & $x$ & $\mathbf{J} \mathbf{s}_{\theta}$ \\
\hline $\mathrm{C} 6\left(180^{\circ} \mathrm{C}\right)$ & $\mathrm{I}, 3715494$ & $=$ & $71,26 \%$ & $x$ & 1,924712 & + & $21,87 \%$ & $x$ & 0 & + & $6,875 \%$ & $x$ & 0 \\
\hline $\mathrm{C} 5\left(190^{\circ} \mathrm{C}\right)$ & $\mathrm{I}, 4003910$ & $=$ & $72,76 \%$ & $x$ & 1,924712 & + & $20,37 \%$ & $x$ & 0 & + & $6,875 \%$ & $x$ & 0 \\
\hline $\mathrm{C} 4\left(200^{\circ} \mathrm{C}\right)$ & I,I 686589 & $=$ & $60,72 \%$ & $x$ & 1,924712 & + & $32,41 \%$ & $x$ & 0 & + & $6,875 \%$ & $x$ & 0 \\
\hline $\mathrm{C} 3\left(210^{\circ} \mathrm{C}\right)$ & I,I25697| & $=$ & $58,49 \%$ & $x$ & 1,924712 & + & $34,64 \%$ & $x$ & 0 & + & $6,875 \%$ & $x$ & 0 \\
\hline $\mathrm{C} 2\left(230^{\circ} \mathrm{C}\right)$ & $\mathrm{I}, 4080497$ & $=$ & $73,16 \%$ & $x$ & 1,924712 & + & $19,97 \%$ & $x$ & 0 & + & $6,875 \%$ & $x$ & 0 \\
\hline $\mathrm{Cl}\left(250^{\circ} \mathrm{C}\right)$ & $\mathrm{I}, 4893254$ & $=$ & $77,38 \%$ & $x$ & 1,924712 & + & $15,75 \%$ & $x$ & 0 & + & $6,875 \%$ & $x$ & 0 \\
\hline TI & $\mathrm{I}, 4 \mathrm{I} 63000$ & $=$ & $73,59 \%$ & $x$ & $1,9247 \mid 2$ & + & $19,54 \%$ & $x$ & 0 & + & $6,875 \%$ & $x$ & 0 \\
\hline $\mathrm{T} 2$ & 1,6114139 & $=$ & $83,72 \%$ & $x$ & I,9247|2 & + & $9,40 \%$ & $x$ & 0 & + & $6,875 \%$ & $x$ & 0 \\
\hline
\end{tabular}

Tabela 8. Teor (porcentagem em peso) de Cromo presente na cementita

\begin{tabular}{cc}
\hline Amostra & \%peso de Cr em $\Theta$ \\
\hline $\mathrm{B} 5\left(190^{\circ} \mathrm{C}-6 \mathrm{~h}\right)$ & 7,18 \\
\hline
\end{tabular}

Tabela 9. Análise metalográfica quantitativa de cementita

\begin{tabular}{ccccc}
\hline Ppm & $\begin{array}{c}\text { Desvio } \\
\text { Padrão }\end{array}$ & \%RA & t & $\begin{array}{c}\mathbf{n} \\
\text { (mínimo) }\end{array}$ \\
\hline $6,875 \%$ & 3,244 & $13,8 \%$ & 2,093 & 2,7 \\
\hline
\end{tabular}

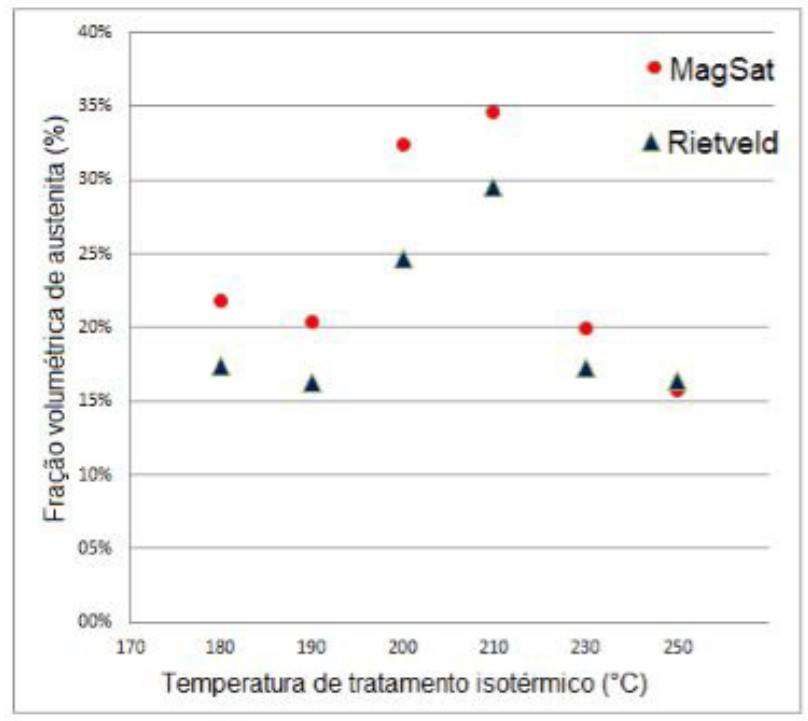

Figura 6. Comparação da fração volumétrica de austenita retida para os métodos de DRX e MagSat.

\section{DISCUSSÕES}

Como a fração de cementita foi estabelecida por um método auxiliar e é constante para ambas as técnicas, 0 comparativo pode ser feito apenas em função da austenita retida. Os resultados são apresentados na Figura 6.
Os resultados são bastante coerentes para as amostras tratadas a $230{ }^{\circ} \mathrm{C}$ e $250{ }^{\circ} \mathrm{C}$. Para as demais amostras, as frações de austenita obtidas por DRX são ligeiramente mais baixas. É importante salientar que, no método de quantificação por saturação magnética, tem-se como amostragem todo o volume da amostra, enquanto para $D R X$, apenas a região próxima à superfície é analisada. A divergência para as amostras tratadas a temperaturas mais baixas pode ser decorrente de eventual transformação da austenita em martensita na superfície durante o lixamento e polimento da amostra na preparação para análise por DRX. Para temperaturas mais baixas o carbono na austenita é menor e, consequentemente, sua estabilidade é menor.

\section{CONCLUSÕES}

A técnica de identificação de fases e quantificação das frações presentes em um aço multifásico através de medidas de saturação magnética aliada às técnicas de microscopia e quantificação por análise metalográfica mostrou resultados coerentes, se comparada à técnica mais usual de DRX. No método proposto, alguns problemas relevantes comuns à técnica de DRX foram mitigados, como textura da amostra, transformação da austenita em martensita durante a preparação, amostragem superficial, etc. Estas vantagens, aliadas ao custo, simplicidade, interpretação de resultados, e facilidade de preparação das amostras faz com que medições por saturação magnética seja uma opção muito interessante e eficaz. Uma análise preliminar matemática, microestrutural, e das propriedades magnéticas dos constituintes presentes no material, porém, é necessária, para que se investigue a real possibilidade de quantificação das fases pelo método. Ainda assim, ligeiros desvios nos resultados foram verificados, e uma análise estatística mais ampla pode ser assunto de um trabalho futuro.

\section{REFERÊNCIAS}

I Speer JG, Rizzo FCA, Matlock DK, Edmonds DV. The "quenching and partitioning" process: background and recent progress. Materials Research. 2005;8:4 I7-423. 
2 Edmonds DV, Speer JG. Martensitic steels with carbide free microstructures containing retained austenite. Materials Science and Technology. 2010;26:386-391.

3 Goldenstein H. Bainita nos aços. In: Bott I, Rios P, Paranhos R. Aços: perspectivas para os próximos 10 anos. Rio de Janeiro: FINEP; 2002. p. 77-88.

4 Bhadeshia HKDH, Edmonds DV. The bainite transformation in a silicon steel. Metallurgical Transactions. A, Physical Metallurgy and Materials Science. 1979;10A:895-907.

5 Caballero FG, Bhadeshia HKDH. Very strong bainite. Solid State and Materials Science. 2004;8:25I-257.

6 Sourmail T, Caballero FG, Garcia-Mateo C, Smanio V, Ziegler C, Kuntz M, et al. Evaluation of potential of high Si high $C$ steel nanostructured bainite for wear and fatigue applications. Materials Science and Technology. 2013;29: I I66-II73.

7 Marcomini JB, Goldenstein H. Nova liga Fe-C-Mn-Si-Cr para rolamentos: curvas de revenimento e fragilização da martensita revenida. Tecnologica em Metalurgia, Materiais e Mineração. 2012;9:324-329.

8 Valdebenito DF. Agregados de ferrita e austenita em aço AISI 52100 modificado com tratamentos isotérmicos [dissertação de mestrado]. São Paulo: Universidade de São Paulo; 2016.

9 Berkowitz AE. Chapter VII: constitution of multiphase alloys. In: Berkowitz AE, Kneller E. Magnetism and metallurgy. New York: Academic Press; 1969. p. 331-363. vol. I.

10 Cullity BD, Graham CD. Introduction to magnetic materials. 2. ed. New Jersey: IEEE Press; 2009.

I I Andrews KW. Empirical formulae for the calculation of some transformation temperatures. Journal of the Iron and Steel Institute. 1965;203:721-727.

I 2 Kagawa A, Okamoto T. Lattice parameters of cementite in Fe-C-X (X $=\mathrm{Cr}, \mathrm{Mn}$, Mo, and Ni) alloys. Transactions JIM. 1979;20:659-666.

I 3 ASTM International. ASTM E-562- I I: standard test method for determining volume fraction by systematic manual point count. United States; 2015.7 p.

Recebido em: 26 Abr. 2016

Aceito em: 14 Set. 2016 